\title{
Bombardieri E, Seregni E, Evangelista L, Chiesa C, Chiti A (Eds.) Clinical Applications of Nuclear Medicine Targeted Therapy. Springer International Publishing Switzerland 2018. ISBN 978-3-319-63067-0
}

\author{
Luigi Mansi ${ }^{1}$
}

Published online: 31 October 2018

(C) Springer-Verlag GmbH Germany, part of Springer Nature 2018

This is an interesting book based on the clinical application of the most recent and innovative nuclear medicine therapies. Authored by internationally well-known specialists with a specific experience in the field, the publication is edited by five Italian experts: four nuclear medicine physicians, Emilio Bombardieri (Humanitas Gavazzeni, Bergamo), Ettore Seregni (National Cancer Research Institute, Milan), Laura Evangelista (Veneto Institute of Oncology IOV-IRCCS, Padua) and Arturo Chiti (Humanitas Rozzano, Milan) and a physicist, Carlo Chiesa (National Cancer Research Institute, Milan). In more than 500 pages, divided into 37 chapters, the authors extensively illustrate the clinical utility of nuclear medicine targeted therapies. The volume is enriched by high-quality figures and didactic tables and by an updated bibliography. The book has been conceived in a specific format in which are presented: 1) diagnosis, 2) chemical properties of the radiopharmaceuticals, 3) clinical indications and practical information, 4) dosimetry, and 5) the current guidelines and the relevance of nuclear medicine in them.

The messages given by the authors are relative to the use of nuclear medicine targeted therapies in some of the neoplasms, such as thyroid, hepatic cancer, neuroendocrine tumors, prostate cancer, and lymphoma, in which clinical results have already been obtained. Furthermore, the last part of the book has been completely dedicated to the new approaches of radiometabolic therapy, such as those using PSMA-based therapy, CXCR4-endoradiotherapy, and new agents for the non-GEP-NET tumors.

The collection of the information provided by the involved international researchers represents a key point for the readers, providing the opportunity to acquire a whole and extensive knowledge in a field in which many patients may be more effectively treated than in the past.

Careful reading of each chapter provides an insight about the diagnosis of benign and malignant conditions. Starting from the information on mechanisms of action, different radiopharmaceuticals are presented, their pharmacokinetic and pharmacodynamic properties being analyzed to provide better understanding of correct clinical indications and their efficacy in specific populations. Moreover, the dosimetric evaluation, comprehensively treated, can help the nuclear medicine physician in the optimization of the therapeutic dose. Finally, a critical analysis of the current international guidelines may assist in improving the inclusion of nuclear medicine targeted therapies in the therapeutic flow-chart of patients affected by benign and malignant tumors.

In my opinion, this book would be helpful to a multidisciplinary vision, considering physicists, radiochemists, and nuclear medicine physicians. The publication can also give significant support to students, residents, and clinicians who are interested in this topic.
Luigi Mansi

mansi.luigi@libero.it

1 Interuniversity Research Center for Sustainability (CIRPS), Naples, Italy 